\title{
Immunologic markers to arrector pili muscle, blister tissue and vessels in a case of bullous eczema herpeticum
}

\author{
Ana Maria Abreu Velez ${ }^{1}$ Carlos Andres Valencia-Yepes ${ }^{2}$, Michael S. Howard ${ }^{1}$ \\ ${ }^{1}$ Georgia Dermatopathology Associates, Atlanta, Georgia, USA, ${ }^{2}$ University of Antioquia, Medellin, Colombia
}

Corresponding author: Ana Maria Abreu Velez, M.D., Ph.D., E-mail: abreuvelez@yahoo.com

Sir,

Eczema herpeticum is an entity usually seen in pediatric and young adult patients, and presents perils of systemic compromise and a ten percent mortality rate $[1,2]$. We describe a 53 year old Caucasian female with a childhood history of atopy who consulted her dermatologist for a sudden presentation of generalized, annular, scaly, itchy, erythematous plaques with some raised ridges and blistering; these lesions were observed concurrent with malaise (Fig. la, black arrow). Our study was performed accordance with the Code of Ethics of the World Medical Association (Declaration of Helsinki); informed consent was obtained. Lesional skin biopsies were taken for hematoxylin and eosin (H\&E) review, direct immunofluorescence (DIF) and immunohistochemistry (IHC) staining. Review of the $H \& E$ sections demonstrated an infectious process, suggestive of a herpes virus infection. Focal areas of the epidermis displayed ballooning of keratinocytes, with margination of cell chromatin and multinucleated cells seen inside an epidermal blister (Fig. 1b, black arrows) (400x). The entire dermis was edematous, including the appendageal structures. A multilevel epidermal blistering process was present. Within the blister lumen, numerous neutrophils, eosinophils and Langerhans histiocytes were also noted. Inflammatory cell debris and acantholytic keratinocytes were also present within the blister lumen. In the subjacent dermis, a superficial, perivascular, mild mixed inflammatory infiltrate was present, featuring numerous lymphocytes, histiocytes, neutrophils and eosinophils. No frank vasculitis was noted.
Our DIF was performed as previously described [3], and demonstrated positivity to the lateral aspects of arrector pili muscles with FITC conjugated anti-human fibrinogen antibodies (Fig. lc, green staining; white arrow). Other findings included IgG (+/-, dermal eccrine glands); IgA (+/-, superficial dermal perivascular); Complement/Clq (+/-, superficial dermal perieccrine); Complement/C3 (+, BMZ granular); Complement/ C5b-9(+/-, superficial dermal perivascular); albumin $(+$, superficial dermal eccrine); and fibrinogen $(+$, superficial dermal interstitial).

For our IHC staining, we utilized CD45, von Willembrand Factor (VWF) and Complement/C4, all from Novocastra (Buffalo Grove, Illinois, USA). We utilized ribosomal protein S6-pS240 phosphorylation site specific (RIBO), and herpes virus I from Santa Cruz Biotechnology (Santa Cruz, California, USA). Our IHC staining was performed as previously described [3]. In Fig. ld, we show positive staining with herpes 1 antibody in the epidermal blister (black arrow), and on upper dermal vessels and inflammatory cells (blue arrow) (100X). Double IHC staining using CD15, myeloperoxidase, CD45, RIBO, Complement/ $\mathrm{C} 4$ and Von Willembrand factor revealed a similar pattern of positivity as that seen with the herpes virus 1 staining. In Fig. le, we show a double color IHC with Complement/C4 positive in the blister (brown staining; blue arrow), and RIBO in the epidermis surrounding the blister (red staining; black arrows) (200X), in the epidermal granular layer above the blister and on dermal inflammatory cells below the blister(red staining; black arrows) (200X).

\footnotetext{
How to cite this article: Velez AMA, Valencia-Yepes CA, Howard MS. Immunologic markers to arrector pili muscle, blister tissue and vessels in a case of bullous eczema herpeticum. Our Dermatol Online. 2017;8(3):361-363.

Submission: 10.10.2016; Acceptance: 01.02.2017

DOI: 10.7241 /ourd.20173.105
} 


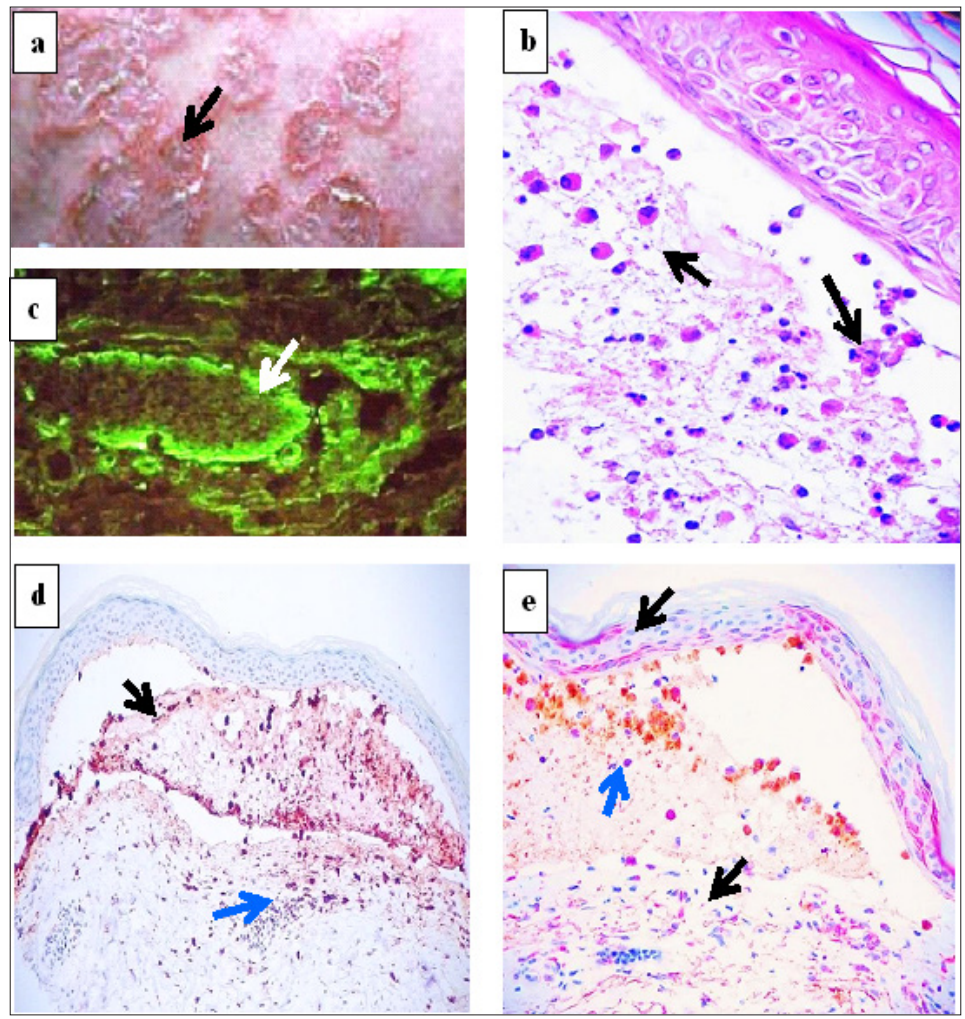

Figure 1: a. display some annular, scaly, itchy, erythematous plaques with some raised ridges and blistering lesions (black arrow). b. $\mathrm{H} \& \mathrm{E}$ shows focal areas of the epidermis displayed ballooning of keratinocytes, with margination of cell chromatin and multinucleated cells seen inside an epidermal blister (black arrows). c. The DIF demonstrated positivity to the lateral aspects of arrector pili muscles with FITC conjugated antihuman fibrinogen antibodies (green staining; white arrow). d. IHC stain show positive staining with herpes 1 antibody in the epidermal blister (black arrow), and on upper dermal vessels and inflammatory cells (blue arrow) (100X). e. Double IHC staining using shows a double color IHC with Complement/C4 positive in the blister (brown staining; blue arrow), and RIBO in the epidermis surrounding the blister (red staining; black arrows) (200X), in the epidermal granular layer above the blister and on dermal inflammatory cells below the blister (fuchsia staining; black arrows)(200X).

Eczema herpeticum is an acute dermatosis, caused by herpes simplex virus type 1 in atopic dermatitis patients. It presents primarily in younger patients, and is considered a dermatologic emergency [1,2]. Eczema herpeticum is rare, and has been associated with r $501 \mathrm{x}$ and 2282del4 filaggrin null mutations [4]. Although the disorder is uncommon in adults, it should be considered in patients with a clinical history of atopic dermatitis who suddenly present with circinate and erythematous blisters with malaise.

We report this case because of the lack of cases in the medical literature showing IHC and DIF immunologic markers in this disorder. We document the presence of CD15, myeloperoxidase and CD45 positive cells in the blister and around subjacent dermal vessels, as well as RIBO, and Complement/C4. Neutrophils, T lymphocytes, endothelial cells, complement, fibrinogen and immunoglobulins respond to the viral infection, and produce intracellular cell signaling indicated by our positive staining for RIBO [5].
Based on our findings, we speculate that the virus seems to affect the epidermis and the subjacent dermal vessels, and arrector pili muscles that are rich in tight cell junctions [3]. Herpes viruses are believed to enter cells through multiple structural sites such as tight, adherens and occludens junctions present in vessels; these cell junctions are also present in arrector pili muscles.

The immune response seen here indicates a complex interaction between the host and the virus. Since young atopic patients are prone to viral infections, is possible that our patient's previous childhood exposure to viruses may have played a role in the strong immune reactivity seen in her current adult case.

\section{ACKNOWLEDGEMENT}

Jonathan S. Jones, HT (ASCP) at Georgia Dermatopathology Associates provided excellent technical assistance. 


\section{ABBREVIATIONS}

Hematoxylin and eosin (H\&E), immunohistochemistry (IHC), direct immunofluorescence (DIF), von Willembrand Factor (VWF), 4', 6-diamidino-2phenylindole (DAPI), ribosomal protein S6-ps240 (RIBO).

\section{REFERENCES}

1. Blanter M, Vickers J, Russo M, Safai B. Eczema Herpeticum: Would You Know It If You Saw It? Pediatr Emerg Care. 2015;31:586-8.

2. Wollenberg A, Zoch C, Wetzel S, Plewig G, Przybilla B. Predisposing factors and clinical features of eczema herpeticum: a retrospective analysis of 100 cases. J Am Acad Dermatol. 2003;49:198-205.
3. Abreu-Velez AM, Klein AD, Howard MS. Junctional adhesion molecule overexpression in Kaposi varicelliform eruption skin lesions as a possible herpes virus entry site. N Am J Med Sci. 2010;2:433-7.

4. Valdés-Rodríguez R, Saavedra-Alanís VM, Islas-Aguilar MA, TorresÁlvarez B. [Eczema herpeticum in a patient with atopic dermatitis, carrying r501x and 2282del4 filaggrin null mutations]. Gac Med Mex. 2015;151:252-5.

5. Stoermer KA, Morrison TE. Complement and viral pathogenesis. Virology. 2011;411:362-73.

Copyright by Ana Maria Abreu Velez, et al. This is an open-access article distributed under the terms of the Creative Commons Attribution License, which permits unrestricted use, distribution, and reproduction in any medium, provided the original author and source are credited.

Source of Support: Georgia Dermatopathology Associates, Atlanta,

Georgia, USA, Conflict of Interest: None declared. 